\title{
Identités narratives et identifications sensibles : itinéraires de migrants franco-cambodgiens de seconde génération à Phnom Penh
}

Narrative Identities and Sensitive Identifications. A Reconstruction of some French Cambodian Second Generation Migrants' Itineraries in Phnom Penh Identidades narrativas e identificación sensible. Reconstrucción de las rutas de migrantes franceses de Camboya de segunda generación en Phnom Penh

\section{Léo Mariani}

\section{OpenEdition}

\section{Journals}

Édition électronique

URL : https://journals.openedition.org/remi/6065

DOI : 10.4000/remi.6065

ISSN : $1777-5418$

Éditeur

Université de Poitiers

Édition imprimée

Date de publication : 1 septembre 2012

Pagination : 89-106

ISBN : 979-10-90426-05-4

ISSN : 0765-0752

Référence électronique

Léo Mariani, «Identités narratives et identifications sensibles : itinéraires de migrants francocambodgiens de seconde génération à Phnom Penh », Revue européenne des migrations internationales [En ligne], vol. 28 - n³ | 2012, mis en ligne le 01 septembre 2015, consulté le 16 avril 2022. URL : http://journals.openedition.org/remi/6065 ; DOI : https://doi.org/10.4000/remi.6065 


\section{Identités narratives et identifications} sensibles:

\section{itinéraires de migrants franco-cambodgiens de seconde génération à Phnom Penh}

\section{Léo Mariani'1}

Des trois pays de l'ancienne Indochine française, le Cambodge est incontestablement celui dont l'histoire contemporaine reste la plus tourmentée, tout entière déformée par le génocide de près de deux millions de personnes perpétré par les Khmers Rouges, entre 1975 et 1979. Cette tragédie, qui semble survenir comme le point d'orgue d'une histoire contemporaine aux dynamiques labiles, façonnée par un contexte international tendu, une situation régionale singulière et un souverain aussi brillant qu'insaisissable, donna lieu à plusieurs vagues d'exode, un mouvement que les élites fuyant la guerre civile, puis la remontée des troupes communistes vers Phnom Penh, avaient initié dès 1970, et qui s'était accéléré dans les mois précédant l'instauration du Kampuchéa Démocratique². Après quatre années d'un obscurantisme total, la prise de Phnom Penh par les forces du voisin et ennemi héréditaire vietnamien, en 1979, provoqua une nouvelle hémorragie de population, sans précédent, vers les camps installés en Thaïlande dans un premier temps, puis à destination de divers pays d'accueil comme la France et les États-Unis dans un second temps. Au total, on dénombrait ainsi environ 50000 réfugiés cambodgiens en France en 1989 (Simon-Barouh, 1989) et, selon I'INSEE, 63284 Français d'origine cambodgienne dix ans plus tard (Nann, 2007).

En 1989, le départ des troupes vietnamiennes du Cambodge suscita la rédaction d'une nouvelle constitution suivie, le 23 octobre 1991, par la signature des accords de paix de Paris qui rétablissaient la souveraineté et l'intégrité du pays. Ces événements successifs occasionnèrent une première vague importante de retours à laquelle participa le souverain légitime, le prince Norodom Sihanouk, jusque-là exilé en Chine, ainsi que des dizaines de milliers de réfugiés rapatriés par I'ONU depuis les camps thaïlandais dans lesquels ils s'étaient réfugiés. Parmi les Cambodgiens qui avaient été en mesure de gagner un pays d'accueil, un certain nombre de membres politisés de la première génération de réfugiés choisirent aussi ce moment pour retrouver leur pays et se ménager une place dans le jeu politique naissant. Lucides quant à l'état encore très précaire du Cambodge, et conscients des risques que leur engagement et leur statut pourraient faire

1 Anthropologue, Chercheur associé à I'URMIS (UMR 205) et au LASMIC (EA 3179) ; leolienne@ hotmail.com

2 Le Kampuchéa Démocratique est le nom donné par les Khmers rouges au Cambodge actuel, la République Khmère (1970-1975) à l'époque de leur prise de pouvoir. 
courir à leurs proches ${ }^{3}$, les Franco-Cambodgiens qui entreprirent cette aventure (principalement des hommes) se résignèrent très souvent à faire le voyage seuls, sans les membres de leur famille restés en France. Depuis la fin des années 1990, et la pacification relative du contexte socio-politique cambodgien, ils sont imités par une population plus hétérogène dont font parfois partie leurs enfants et qui, comme au Laos (Souvannavong, 1997) et au Vietnam (Phong et al., 2000), semble connaître un accroissement régulier. Le présent article expose les résultats préliminaires d'une recherche réalisée entre les mois d'avril et de décembre $2010^{4}$, dans la capitale cambodgienne, auprès de membres de cette population nés en France (issus de la deuxième génération) ou y ayant passé l'essentiel de leur vie ("la génération 1,5 ", Levitt et Waters, 2002). II se base sur un corpus d'entretiens et d'observations recueilli auprès de quinze personnes choisies au hasard (huit hommes et sept femmes).

Parmi mes informateurs, neuf sont nés en France entre 1977 et 1983, trois au Cambodge ou dans les camps thaïlandais en 1982 et 1983, et trois sont nés au Cambodge entre 1966 et 1974. Parmi les six qui ne sont pas nés en France, quatre y sont arrivés avant l'âge de cinq ans, un à six et le plus âgé à neuf. Notons enfin que les ascendances chinoises sont nombreuses dans la population cambodgienne. Dans les cas étudiés, elles ne semblent cependant avoir donné lieu à une transmission que lorsqu'elles suivaient une ligne agnatique. Seules deux des personnes interrogées utilisaient ainsi un dialecte chinois pour communiquer avec leurs parents et s'identifiaient comme "Chinois du Cambodge", marquant de la sorte leur appartenance à une population séculairement implantée dans le pays. Dans les conversations, cependant, ces personnes usaient indifféremment des termes "Cambodgien " et "Khmer " pour qualifier leur double appartenance, même lorsqu'ils ne possédaient aucune ascendance khmère connue.

\section{À propos du positionnement, la dimension sensible de l'ex- périence}

Mes recherches se sont dans un premier temps organisées autour du recueil d'histoires de vie et d'histoires de migration (Bretell, 2003) avec pour unique objectif de situer le départ de France dans une histoire personnelle. Dans un second temps, I'analyse a porté sur l'interprétation de leur expérience par les migrants, la dimension narrative de leur identité (Ricœur, 1985), ainsi que sur des données sociologiques et psychologiques plus objectives que la mise en forme du vécu laissait échapper. J'ai parallèlement essayé de mettre en perspective les informations ainsi obtenues avec des observations plus concrètes du quotidien.

L'hypothèse méthodologique qui guide cette démarche repose sur le refus simultané de plaquer des modèles d'analyse pré-conçus sur la réalité d'une part et de proposer une lecture purement empirique et donc univoque du phénomène étudié d'autre part.

\footnotetext{
3 La situation politique et sociale du Cambodge ne s'est véritablement pacifiée qu'à la fin des années 1990, après la mort de Pol Pot, le leader khmer rouge retranché dans l'Ouest du pays avec ses derniers fidèles, et suite à la mise en place d'importantes mesures visant à désarmer la population. Avant cela, les règlements de comptes et les enlèvements restaient très fréquents à Phnom Penh. Ils touchaient tout particulièrement ceux qui étaient susceptibles de pouvoir payer une rançon. Pour les Cambodgiens de l'étranger, il était donc impossible de se déplacer dans la capitale sans être accompagnés de plusieurs gardes du corps ( $d$ 'autant plus s'ils avaient des proches engagés en politique).

4 Cette recherche a bénéficié du soutien financier et logistique du CKS (Center for Khmer Studies), à Phnom Penh. Je souhaiterais par ailleurs remercier Messieurs Joël Candau et Bernard Sellato pour leurs remarques et suggestions concernant cet article, ainsi que Pascale Hancart-Petitet pour son ultime relecture.
} 
Pour ces raisons, l'élaboration de l'interprétation qui suit prend sa source autant dans ce que les acteurs font que dans ce qu'ils se représentent, dans le sens qu'ils confèrent à leur expérience. Bien que n'ayant jamais cessé de confronter les discours à certains faits plus objectifs, j'ai de cette façon choisi de m'intéresser d'abord aux anthropologies ordinaires, celles que tout un chacun développe au quotidien. Puis, j'ai cherché à replacer ces interprétations dans le contexte de leur apparition, à l'instant anthropologique où chacun, comme le fait l'ethnologue, se met à "lancer des ponts entre le sensible et l'intelligible " (Lévi-Strauss, 1971), donnant forme à ce qui jusque-là n'existait qu'à l'état latent, embedded dans l'expérience. Ce positionnement s'est fait tout seul, à mesure que je prenais conscience du caractère insuffisant des explications posées en termes logiques (les plus fréquentes) pour rendre compte de l'expérience des personnes rencontrées. En effet, et comme le fait remarquer Joël Candau (2011), le privilège qui est accordé au logos dans la compréhension des processus identitaires (par exemple) est manifeste dans la littérature scientifique, laquelle s'attache en général à l'appréhension des " "stratégies identitaires mises en œuvre," "utilisées", "mobilisées,, "développées" ou encore "élaborées" par des "acteurs" " ainsi réduits à leur rationalité alors que l'identité est aussi question sensible, liée à ce que Jean-Paul Sartre nommait la "conscience irréfléchie " des choses (en opposition à la " conscience réfléchie " du cogito réflexif) et qu'on retrouve sous des formes voisines dans toute la phénoménologie, notamment dans le concept de "pré-réflexibilité " cher à Maurice Merleau-Ponty et ré-approprié plus tard par Pierre Bourdieu. Cet article repose donc sur cette idée simple que la conscience ne provient pas de rien, mais qu'elle se détache d'un fond pré-réflexif. L'argumentation a ensuite pour objectif de présenter quelques éléments susceptibles de donner corps à ce "fond " puis, enfin, d'évoquer son essentialisation ponctuelle par les personnes rencontrées comme par l'ethnologue.

II est certain qu'en prenant la dimension sensible de l'existence pour principal objet, cet article risque de donner l'impression de minimiser du même coup la contribution du calcul et de la rationalité au déploiement des itinéraires. On doit toutefois l'envisager comme un préalable non exclusif sur lequel des analyses en termes plus conventionnels pourront par la suite être développées. Il sera donc ici question du cadre existentiel général dans lequel les personnes que j'ai rencontrées paraissent évoluer.

La posture adoptée au moment du terrain est donc fondamentalement rattachée, dans sa première phase, à l'instauration d'un cadre global défini par le recueil d'histoires de vie orientées autour de la question du retour. L'accumulation des entretiens, progressivement complétée par des observations, a ensuite d'elle-même fait ressortir des lignes de forces qui, dans les premiers moments, étaient loin d'être perceptibles. Toutes les discussions qui servent de base de réflexion à cet article ont par conséquent été réalisées selon les mêmes principes méthodologiques. Les objectifs de l'entretien étaient à chaque fois présentés en mettant d'une part l'accent sur l'objectif principal de la recherche : comprendre les raisons du retour ainsi que ses conséquences, et d'autre part sur ma volonté de replacer cet événement dans un cadre plus large où I'histoire des parents, de leur départ du Cambodge, était censée faire office d'amorce. Ensuite, une grande liberté était laissée aux personnes interviewées pour présenter leur itinéraire français, leur départ puis leur arrivée au Cambodge.

La mise en place d'une telle définition de l'interaction n'a pas été sans provoquer, parfois, des incompréhensions, car l'a priori méthodologique de l'ethnologue, pour lequel I'installation à Phnom Penh ne pouvait être saisie qu'à I'horizon d'une histoire plus globale, ne faisait pas toujours sens aux yeux de ses informateurs. Ce décalage fut parfois d'autant plus important que l'expérience migratoire est presque systématiquement concentrée dans le moment de "l'intégration " par le sens commun comme par les 
lecteurs légitimes du phénomène que sont les médias ${ }^{5}$ ou encore les politiques. Pourtant, si trouver une place dans la société d'accueil est à n'en pas douter une étape importante du processus migratoire, ce dernier peut d'autant moins y être réduit dans le cas de cette étude que, parmi les personnes rencontrées, beaucoup semblent y être parvenues, corrélant précisément cette réussite à l'abandon d'un objectif d'intégration implicitement compris et représenté en termes assimilationnistes.

Ainsi, l'arrivée au Cambodge suscite-t-elle fréquemment l'espoir de passer inaperçu, " de se fondre dans la masse "; une aspiration que cristallise et résume très bien la problématique de l'apparence physique qui, en France, était perçue comme indépassable en dernière instance. $\mathrm{Si}$, dans les premiers moments, la croyance en une intégration facilitée au sein du spectre phénotypique asiatique ${ }^{6}$ demeure donc très présente, elle se trouve toutefois progressivement contrariée à mesure que les migrants prennent conscience de l'imprégnation culturelle de leur corps, de leurs mouvements, et de l'étrangeté qu'elle génère malgré tout et en toute circonstance. Cette observation, qui est particulièrement récurrente dans les témoignages, a plusieurs conséquences. Elle permet notamment de montrer que le processus de classification des migrants par la société d'accueil est très loin de se limiter à des critères esthétiques associés à des déterminants biologiques, mais procède aussi à partir de données culturelles implicites qui ont trait, conformément à l'intuition maussienne (1934), à la mise en forme du corps par la culture d'origine. Cette dimension (sensible) de la perception des individus étant soustraite à toute conscience immédiate, elle échappe le plus souvent aux formulations les plus courantes de la différence, qui se bornent à l'exprimer de façon très sommaire, selon des stéréotypes de sens commun. Lors des premiers contacts, les Cambodgiens identifient ainsi souvent ces migrants à des "Coréens ", des "Japonais ", ou encore à des "Philippins" (cf. ci-dessous). Ils témoignent de la sorte de la reconnaissance immédiate du différent (culturel) dans le même (biologique), de la perception d'une étrangeté mal circonscrite qui, parce qu'elle contredit tous les attendus sensoriels et ne correspond à aucune catégorie préalable, ne peut être fixée et explicitée (a minima s'entend) que par I'assignation d'une identité asiatique distincte. À bien y regarder, la diversité phénotypique de Phnom Penh, dont la population est très largement métissée, offre pourtant de nombreuses alternatives de classement et les choix réalisés montrent essentiellement que les personnes rencontrées ont d'abord été identifiées comme non-Cambodgiennes plutôt que comme distinctes parmi les Cambodgiens (comme peuvent l'être par exemple les "Chinois du Cambodge "). Même lorsqu'ils ne sont pas nécessairement attribués à dessein, les stéréotypes privent par définition I' " autre " de certains attributs (Herzfeld, 1992 : 67).

Du point de vue des migrants, cette forme de stigmatisation " instinctive " semble avoir des échos variables selon que les personnes estiment que leurs caractéristiques physiques coïncident ou non avec les canons khmers, tels qu'ils semblent assez consensuellement transmis dans le pays d'origine. Ainsi, même si ces archétypes ne possèdent qu'une réalité très relative à Phnom Penh, les membres de la première catégorie semblent généralement plus affectés par la rencontre, car la certitude de leur conformité avec l'idéal khmer leur a été historiquement attribuée en France, par leurs parents comme par les membres de la communauté. En l'absence des garde-fous qu'une altérité relative, mais incorporée, procure à ceux parmi mes informateurs qui n'ont jamais été associés à

\footnotetext{
5 Voir par exemple le dossier que le Cambodge Soir Hebdo numéro 148 (2 au 8 septembre 2010) consacre au sujet ou encore l'article proposé sur le site d'information Ka-set (http://ka-set. info/actualites/cultures-et-societe/cambodge-diaspora-cambodgien-etranger-khmer-retour-identite-090911.html).

6 Celui-ci est très large à Phnom Penh.
} 
cette représentation (à qui on a au contraire enseigné qu'ils ne ressemblaient pas à des Khmers7), ils se trouvent confrontés d'une façon la plus radicale qui soit à la mise en perspective de leur différence et, partant, à la profondeur de leur singularité. Dès lors, le face-à-face avec la société cambodgienne peut prendre une tournure très désagréable, comme dans le cas de Sopha ${ }^{8}$ que son entourage avait toujours assurée de "l'authenticité " de ses traits - " mes connaissances et aussi des anciens, ils m'ont dit : mais tu ressembles vraiment, vraiment aux Khmers d'avant quoi. T'as les traits, les yeux, la bouche, la couleur, enfin tout "-, mais qui, en contexte, est sans arrêt remise devant l'évidence de son altérité. Malgré une apparence assurément concordante avec une idéologie dominante et consensuelle, sur laquelle elle pensait pouvoir s'appuyer pour " s'intégrer ", une personne sur deux au moins s'adresse donc directement à elle en anglais, confirmant ainsi que le phénomène de discrimination décrit engage plusieurs niveaux de perception, même si ceux-ci sont ensuite traduits en termes essentiellement physiologiques. À ce propos, Sopha ne semble jamais avoir été identifiée comme "Coréenne " ou "Japonaise ", mais plutôt comme "Philippine " ou "Indonésienne ", des signifiants pour le moins flottants si l'on considère l'étendue des archipels concernés, mais qui suggèrent néanmoins des types physiques plus proches. Les termes utilisés, s'ils marquent ainsi la reconnaissance d'une certaine proximité physique, désignent tout de même Sopha comme une étrangère.

Peu différenciés du point de vue de leurs traits physiques, les migrants de l'étude le sont corporellement. Cette situation est, comme nous le verrons, à peu près l'inverse de celle qui était la leur en France où, dans la plupart des cas étudiés, seule l'apparence physique semblait parfois encore contrarier une assimilation totale et permanente au sens où l'entendent l'idéologie et l'État français. Dans les faits en tous cas, le fantasme d'une assimilation à la société cambodgienne semble rapidement abandonné, l'intégration se réalisant plutôt de façon alternative, par acceptation des différences.

\section{Processus identitaire et cadre général de la migration}

Si la pertinence du cadre d'entretien proposé n'a donc pas toujours convaincu les personnes interrogées, elle est pourtant apparue comme évidente pour une large partie d'entre elles : parce qu'elles l'avaient déjà intégrée à leur réflexion pour certaines et/ou parce que son irruption fut directement à l'origine de la formation et de la formulation de liens biographiques signifiants et intelligibles. Ainsi, Vannak interprète-t-il de sa propre initiative les deux premières années de sa vie, qu'il a passées au Cambodge avec ses grands-parents alors que ses parents étaient étudiants en France, comme fondatrices dans son projet de retour. Au fil de la discussion, le discours interprétatif laisse même place à la formulation d'un lien de causalité explicite :

" Je pense que c'est une période qui m'a beaucoup marquée. Et d'ailleurs, j'ai interrogé ma mère et mes grands-parents sur mes réactions après le... après mon retour en France... et ça a été assez violent finalement pour moi. Ç'a été... Comme disait ma mère, quand je suis arrivé... quand mes grands-parents m'ont amené, en décembre 74 en France... il paraît que je n'ai plus parlé pendant une semaine. J'étais terrassé par le chagrin [...]. Mes grands-parents m'ont amené en France et après, ils sont repartis au Cambodge... et donc voilà... ça ça m'a pas mal marqué et je me souviens quand même d'une très grande tristesse, tristesse d'avoir quitté le pays... [...]. J'ai été élevé un peu si tu veux comme un petit prince ici [au Cambodge]. Et je pense que la douceur de vivre a dû me marquer.

Et... en fait, je connaissais pas mon père et ma mère. Je suis arrivé en France, je les connaissais

7 Ce qui ne les empêche nullement d'employer le terme "Khmer " pour se définir dans la mesure où la différence entre "Khmer " et "Cambodgien " n’est quasiment jamais marquée.

8 Tous les prénoms ont été modifiés. 
pas! Donc je les appelais par leur prénom. Et le départ de mon grand-père... enfin le départ de mes grands-parents, quand ils m'ont amené en France et qu'ils sont repartis, ça m'a beaucoup, beaucoup marqué. Et... et voilà. Bon je te raconte ça parce que je pense que ça a été... je pense que c'est fondateur dans cette... dans cette envie de revenir, enfin, de venir vivre au Cambodge ".

Très marqué dans ce passage, le processus de construction narrative de l'identité se déploie en toute clarté, le narrateur articulant explicitement un point de vue introspectif et conceptuel d'adulte avec des événements dont il est peu probable qu'il se souvienne véritablement ni même qu'il ait pu les éprouver dans les termes exposés. Connaissance et description de soi se conjuguent donc ici au sein d'une même interprétation. Malgré les spécificités de l'expérience et du point de vue de Vannak, son récit permet par conséquent de mettre le doigt sur la question des raisons du retour qui, chez les personnes interrogées, semblent presque toujours liées à des problématiques identitaires alors qu'elles ne sont que plus secondairement rapportées et rapportables à des questions pratiques, politiques ou familiales. Mon hypothèse ici est que le processus de retour est, dans le cas de la population étudiée au moins, intimement et même organiquement lié à un processus identitaire complexe dont le rythme et la dramaturgie sont finalement plutôt constants malgré la grande diversité des expériences. Ce processus, que les explications les plus courantes de la littérature scientifique peinent à éclairer, soit qu'elles l'ignorent soit qu'elles n'y prêtent pas attention ${ }^{9}$, connaît différentes étapes. II est plus ou moins conscientisé, est parfois influencé par des paramètres plus pragmatiques qui le précipitent, l'orientent ou le freinent, mais il ne se réduit jamais à aucun d'eux, ni même à leur somme. La migration de retour étudiée est en réalité très personnelle ce qui, comme nous le verrons, peut être relié aux spécificités de l'histoire cambodgienne, mais aussi, semblet-il, à des constantes sociologiques d'apparence contradictoire avec le besoin de quitter la France pour un pays presque inconnu.

\section{Intégration et assimilation, l'expérience française}

Parmi les caractéristiques les mieux partagées par mes informateurs, une semble quasiment faire l'unanimité. Elle a trait au niveau d'intégration en France, que la plupart interprètent comme bon voire excellent. Ce pays est ainsi perçu de façon plutôt positive, comme un lieu d'enracinement où la majorité aimerait un jour retourner vivre même si les difficultés relatives à la recherche d'un emploi ainsi qu'à un quotidien plus stressant et surtout plus cloisonnant (où la liberté d'initiative est plus restreinte) sont perçues comme de sérieuses entraves à ce projet.

Au vu des risques que l'entretien de style " récit de vie ", utilisé dans le cadre de cette recherche, fait courir à la qualité des informations recueillies, souvent lissées a posteriori (Bourdieu, 1986), on pourrait être spontanément tenté de relativiser ce ressenti subjectif d'une intégration française réussie. On observe pourtant qu'il est confirmé par un faisceau de données objectives qui, comme nous allons y revenir, placent les personnes de I'enquête dans la catégorie des immigrations réussies au sens où l'entendent l'idéologie et l'État français, voire dans un schéma de réussite moins spécifique, propre aux sociétés occidentales. Si tant est que, comme proposé plus haut, on concède par ailleurs que les sentiments d'identité et d'altérité ne reposent pas seulement sur des considérations réfléchies, mais également sur une réalité sensible pré-réflexive, ces arguments tendent de surcroît à être confortés par la facilité avec laquelle la communication proxémique et kinésique s'est le plus souvent établie entre l'ethnologue et ses informateurs. Mis en

\footnotetext{
9 Beaucoup de travaux continuent ainsi plus ou moins de prendre pour base la typologie établie par Cerase (1974), qui distingue quatre types de migrants de retour selon leurs aspirations, leurs attentes et leurs besoins, mais qui n'avance aucune motivation identitaire.
} 
relation et objectivé, le corps (entendu au sens large) de l'observateur livre en effet des sensations qui, en permettant de mettre les discours en perspective avec des ressentis, sont susceptibles d'offrir un complément d'informations. Dans cette hypothèse, le niveau de connivence interactionnelle et sensible de l'ethnologue avec ses informateurs, qui fut le plus souvent spontané, est parfaitement cohérent avec l'évaluation subjective que ces derniers se faisaient de leur intégration en France.

Quoi qu'il en soit, les informations recueillies montrent que ceux qui étaient déjà insérés dans la vie active avant leur départ jouissaient tous ou presque d'une situation professionnelle, financière et sociale confortable; souvent plus intéressante d'ailleurs que celle de leur arrivée au Cambodge. Du point de vue des caractéristiques sociologiques les plus objectives, cette réussite semble d'abord devoir être associée à un niveau d'étude élevé (onze personnes sur les quinze ont un niveau bac+4 ou 5 et deux un niveau bac+2) qui, contrairement à ce que la raison utilitariste pourrait laisser entendre, n'a pratiquement jamais été à l'origine de la décision de départ. Seules deux personnes du groupe étudié paraissent ainsi avoir fait directement entrer leurs compétences en ligne de compte dans leur choix d'installation au Cambodge. Pour les autres, et comme nous y reviendrons plus loin, le départ de France est la plupart du temps advenu de façon plutôt fortuite, répondant à une opportunité ou, très souvent, à " un coup de tête ", ce qui n'empêche pas en revanche que la perception des avantages liés au diplôme et à l'expérience ait ensuite joué un rôle déterminant dans la prolongation du séjour cambodgien. La mise en relation du niveau d'étude (et au-delà du niveau d'assimilation) avec le départ est donc d'autant plus intéressante à réaliser que c'est la régularité des observations et non un rapport de causalité explicite qui suggère leur corrélation. Le choix d'appréhender le phénomène de retour comme un processus identitaire plus global permet alors de comprendre cette coïncidence de façon complexe, sans nécessairement la réduire à un rapport simple de cause/conséquence.

De fait, les similitudes qui viennent d'être évoquées sont doublées d'analogies concernant les origines géographiques en France et surtout, mais c'est lié, demeurent généralement associées à une forme de marginalité vis-à-vis des autres immigrés franco-cambodgiens. On observe ainsi deux choses : d'abord que les individus venant de petites villes de province où la concentration d'immigrants asiatiques est faible sont très représentés relativement à ceux qui ont grandi là où le niveau d'immigration cambodgienne est le plus fort, en région parisienne ou dans certaines grandes villes notamment. Surtout, quelle que soit leur origine géographique, tous ou quasiment font part d'une situation d'isolement relatif vis-à-vis des communautés cambodgiennes locales (lorsqu'elles existaient), insistant dans plusieurs cas sur le rôle que cette distance a pu jouer dans la réussite de leur intégration française. Comme le souligne Sopheap, I'influence des parents fut souvent décisive dans ce choix :

"À Toulouse, il y avait plein de Cambodgiens. Et en fait, là on s'est rendu compte vraiment de l'éducation que les parents apportaient aux enfants. Donc j'allais finir... C'est pas qu'elle les aime pas ses amis ma mère tu vois, au contraire. Elle les fréquente mais elle ne voulait pas, en fait, que

j'aie cette vision de la vie. Parce que tu sais, quand tu restes en groupe, tu sais les groupes, tu sais comment c'est? [acquiescement] Ben voilà. Donc on est allé à quatre-vingts kilomètres de Toulouse. C'est là que j'ai grandi. Et après le bac, je suis revenue à Toulouse. Mais, avec une vision à la française. J'ai pas vécu avec des Khmers du tout quoi. C'est pas loin quatre-vingts bornes mais franchement, tout de suite, t'es coupé de la communauté quoi. Donc... Bon voilà, j'ai fait mes études à Toulouse et alors, bizarrement, je revois ces filles mais comme s'il y avait un fossé entre nous quoi. On a grandi ensemble, mais juste pour les fêtes religieuses, donc c'est une après-midi tous les ans ". 
L'isolement social et/ou géographique s'impose donc comme un élément déterminant dans les parcours étudiés parce qu'il semble avoir favorisé l'adaptation puis l'assimilation à un environnement où les références au Cambodge étaient par la force des choses quasiment réduites aux circonstances familiales et à quelques événements communautaires ponctuels. De telles circonstances impliquent par conséquent de s'intéresser à la question de la transmission entre générations, laquelle occupe d'ailleurs abondamment les témoignages. On observe ainsi que si la densité des liens affectifs qui innervaient le tissu familial semble ressortir de façon très nette, l'absence de communication et le mutisme des parents reviennent eux aussi comme un leitmotiv que ni l'âge, ni le retour au Cambodge ne semblent avoir été en mesure de contrarier. Paradoxalement, et comme le mentionne Loumsarith à propos de la question du racisme, cette situation n'a pas toujours eu que des effets " négatifs" :

"Euh racisme, après c'est pas du racisme, ça reste de l'ignorance tu sais... C'est... C'est comme on était à la campagne ben t'es le Chinois, le Chintoc quoi. Tu vois. Mais va essayer d'expliquer à des gars de la campagne le Cambodge. Qu'il y a un pays qui s'appelle le Cambodge. Où moi en plus, j'étais pas du tout curieux. Tu vois. Comme j'avais rien en face de moi tu vois. Un gars peut-être qui a vécu dans une... plus en communauté, il a plus quelque chose peut-être devant lui. Il a un miroir, peut-être, qui reflète, où il y a du répondant au niveau de la culture. Moi j'avais rien, y avait juste mes parents. Et mes parents on parlait pas, tu vois".

Les ressorts d'une telle absence de communication sont certainement complexes à dénouer, mais les personnes interrogées les associent essentiellement à un réflexe protecteur d'une part et, d'autre part, à un particularisme " culturel " qui soumet l'expression des sentiments à un contrôle permanent. On peut penser que ces arguments doivent en outre être mis en relation au sein d'un écheveau de causes qui se noue autour d'une histoire personnelle difficile (la plupart des parents ont perdu des proches, voire des enfants parfois) ainsi que d'une volonté somme toute logique de créer les conditions d'un nouveau départ, pour soi comme pour les plus jeunes, le tout dans les circonstances pénibles de l'immigration en France et en l'absence de toute autre référence générationnelle (les grands-parents étant le plus souvent restés au Cambodge). Jusqu'à la période de l'adolescence au moins, les témoignages soulignent ainsi un manque d'intérêt manifeste pour les origines cambodgiennes, faisant pour certains aussi référence à une forme de résignation que l'initiation à des " coutumes qui étaient apparemment différentes de celles du reste des Français ", mais que les parents " n'expliquaient pas ", a contribué à nourrir. $C^{\prime}$ est de fait dans une réalité plutôt immédiate, ancrée dans le présent, que les migrants de mon enquête semblent avoir passé leurs années d'enfance, intégrant des données liées à la culture cambodgienne par habitude, par imitation et par imprégnation (à ce propos, voir aussi Guillou, 2001 ; Nann, 2009), " par corps " donc selon la belle expression de Pierre Bourdieu, mais sans jamais véritablement leur attribuer de statut spécifique.

II faut enfin souligner que parmi les autres éléments qui ont contribué à faciliter l'adaptation des enfants, le contexte politique et émotionnel qui présidait à l'accueil des réfugiés cambodgiens en France a lui aussi probablement joué un rôle décisif. Ainsi, alors que la sollicitude des membres des réseaux associatifs, et religieux pour certains, embarrassait bien souvent les adultes, elle semble avoir donné lieu à une interprétation résolument plus spontanée de la part des jeunes enfants. Rares sont ceux qui, par conséquent, ont pu éprouver en conscience le sentiment de honte et d'obligation mêlées avec lequel leurs aînés devaient composer. Bien au contraire, la découverte et l'exploration du nouvel univers qui s'offrait à eux, facilitées par les attentions dont ils faisaient l'objet, semblent dans plusieurs cas avoir suscité l'épanouissement d'une forme d'innocence et de curiosité propres à l'enfance. 
Bien entendu, ces circonstances plutôt favorables n'ont pas complètement empêché un certain apprentissage de la différence de se faire. Ici aussi, il transparaît que les situations se distribuent peu ou prou selon les deux types d'agencements sociologiques et géographiques évoqués plus haut. Dans le premier cas de figure, on retrouve ainsi les personnes qui ont grandi dans de petites ou moyennes villes de province où le niveau d'immigration (notamment cambodgien) était faible. Curieusement, celles-ci décrivent une certaine indifférence à l'égard de remarques racistes qui, selon elles, n'étaient pas si fréquentes et relevaient plutôt d'une forme d'ignorance que d'un " vrai racisme ". Aussi, ils sont par exemple nombreux à avoir bien toléré la catégorisation de "Chintoc" lorsqu'elle leur était imposée, comme d'autres parmi leurs camarades d'école non marqués par leurs origines s'appropriaient vaille que vaille celles de " gros " ou encore de " laid ". C'est en effet un élément bien établi par les linguistes et les micro-interactionistes que, du point de vue du groupe, la reconnaissance passe principalement par l'acceptation de ses règles et l'appropriation de ses codes, aussi idiots et contraignants soient-ils. Pour que la communication au sens large soit possible et se perpétue, elle se doit ainsi de reposer sur un minimum de coopération et de consensus, lesquels en constituent même la condition nécessaire. Bien évidemment, certaines expériences se démarquent de la norme ${ }^{10}$, mais elles inclinent toutefois à en confirmer la prégnance parce qu'elles sont justement liées au refus du rôle attribué par le groupe, ce qui, dans les situations qui m'ont été communiquées, semble avoir accentué le rejet. Ainsi, si cette dynamique d'agrégation ou d'exclusion est finalement assez binaire, elle n'en reste pas moins complexe dans la mesure où les dynamiques propres au groupe, très imprévisibles, contribuent également à l'influencer. C'est ce que montre bien I'analyse de Rotana, dont la singularité s'est immédiatement trouvée valorisée :

" J'étais plutôt dans une école où y avait... Y avait même pas un Asiatique en fait. J'étais le seul Asiatique donc... Quand t'es le seul Asiatique, t'as pas de discrimination. T'es plutôt le mec différent mais dans le bon sens du terme. Dans le sens positif. Si par exemple tu arrives dans une école avec un groupe d'Asiatiques, tu vas faire peur aux gens. C'est-à-dire... Dans cette classe, il y a dix Asiatiques. C'est ce groupe d'Asiatiques tu vois. C'est cette force qui va faire peur aux autres et qui va créer peut-être des côtés un peu raciaux, etc., etc. Oh, c'est ce groupe! Oh ce sont ces "Asiats"! Oh ce sont ces nouveaux gars qui arrivent dans notre pays ! Tu vois. Mais moi j'ai jamais ressenti ça parce que j'étais plutôt dans une école où j'étais le seul Asiatique. Donc on voulait plus m'aider que de me dire oh ! Qu'est-ce qu'il fait là ? ".

La deuxième tendance de différenciation qui semble se dessiner est à peu près inverse de celle qui vient d'être présentée. Elle concerne ceux qui ont grandi dans un contexte de mixité sociale plus important, principalement en région parisienne ou dans certaines grandes villes de province. Dans ces circonstances, le phénomène de discrimination était, comme on peut s'en douter, très courant. II doit cependant être rapporté à la spécificité de contextes où les stéréotypes de "Chintoc ", "d'Asiat ", de " black ", etc. sont communément mobilisés dans la définition de "l'autre ", et où la discrimination s'applique alors en quelque sorte sans discrimination.

Soit, donc, les migrants de l'enquête ont grandi dans une relative situation d'isolement et semblent ainsi avoir expérimenté leur différence de façon plutôt positive, ou du moins sans nécessairement lui accorder plus d'importance qu'à d'autres signes physiologiques distinctifs, soit la mixité sociale de leur lieu de vie a par la force des choses rendu cette différence plutôt commune et donc somme toute assez normale.

10 Surtout parmi les filles pour qui il est vrai qu'une forme supplémentaire de discrimination, liée au sexe, venait probablement s'ajouter à la perception de la différence physique. 
Bien souvent, la question des origines cambodgiennes a été de la sorte assez facilement éludée jusqu'à l'adolescence au moins, ce qui se traduit aussi par le fait qu'aucune des personnes interrogées $n^{\prime}$ ait entretenu de relations particulières avec d'autres FrancoCambodgiens avant un âge plus avancé et que seules trois parlaient khmer avec leurs parents (une autre dans un dialecte chinois) alors même que ces derniers les sollicitaient presque systématiquement dans cette langue. L'adolescence s'inscrit ensuite dans un schéma plutôt classique de quête identitaire où les origines sociales et/ou culturelles suscitent un positionnement radical de rejet ou, au contraire, d'adhésion. Les témoignages montrent alors que l'idéologie universaliste française a pu parfois procurer une véritable échappatoire en favorisant, vis-à-vis des autres autant que pour soi, l'expression d'une subjectivité propre et donc la formulation d'une définition personnelle positive. C'est le cas de Sébastien dont les origines chinoises ajoutaient à la complexité de la situation :

"Quand je disais Cambodge, c'était Cambodge? Oh les boat people, les Khmers rouges, les camps, les machins! Alors je me suis créé un imaginaire. En plus moi je savais rien parce que mes parents ne parlaient pas de ça. Donc systématiquement c'était Pol Pot et tout ça. Donc moi ça m'a un peu saoulé [...]. Les Chinois m'ont toujours dit mais tu ne parles pas mandarin, tu ne viens pas

de Chine, n'importe quoi, t'es pas Chinois. Les Khmers disaient t'es pas Khmer, les Français t'es pas Français. Donc au final, dans les trois options, j'ai gardé le côté français. L'intégration républicaine [...]. Je me suis dit cette idée de on est tous Français, on est tous égaux, on a beau être noir,

Maghrébin, c'est génial. Je pense que ça c'est un peu marqué au niveau de l'adolescence quoi.

Peut-être que je... Déjà au niveau de l'enfance, y avait pas de... comment dire... Transmission au niveau de... De l'histoire de mes parents tout simplement, de leur famille, de leur culture d'origine, mis à part la nourriture cambodgienne et il faut aller à la pagode pour aller prier... On savait même

pas pourquoi on le faisait à la limite. On le faisait parce qu'il fallait le faire. Voilà, et puis moi, la pagode, c'était les jeux de cartes dans la pelouse, c'était les ballons c'est tout quoi. Et même des coutumes qui étaient apparemment différentes du reste des Français, ils ne les expliquaient pas...

Comme moi je m'en suis désintéressé encore plus au niveau de l'adolescence, ce qui fait que à tout cet âge-là, jusqu'à ma vie d'adulte, j'ai eu... aucun intérêt comme je te l'ai dit ".

Cette adhésion plus ou moins choisie au modèle républicain d'intégration est à mettre en perspective avec un positionnement contraire tout aussi radical quoique moins courant :

"À un moment, en ayant la vingtaine, j'ai eu une petite crise d'identité où je ne sortais qu'avec des copains cambodgiens, où j'allais à des fêtes... cambodgiennes au moment du Nouvel An. J'avais envie d'un retour aux sources. Et puis après je me suis aperçue... que j'étais peut-être un peu différente de mes amis cambodgiens. Donc je suis revenue à une autre... à une autre façon de vivre ".

C'est en réalité dans les années qui suivent l'adolescence que le positionnement a en général opéré son premier tournant sensible, se faisant moins radical et surtout plus pragmatique. Au cours de cette période, qui coïncide dans plus de la moitié des cas avec une expérience universitaire, il apparaît clairement que l'influence des sollicitations extérieures, tant amicales qu'académiques, a joué un rôle déterminant dans le cheminement progressif vers une identité cambodgienne jusqu'alors ignorée ou rejetée. Certains témoignent ainsi de l'état de frustration dans lequel les sollicitations croissantes de leur entourage les mettaient, leur imposant une curiosité qu'ils n'étaient pas en mesure de satisfaire et qu'ils ne pouvaient pourtant pas fuir indéfiniment. D'autres racontent la jalousie et la déception éprouvées au contact d'amis revenus de vacances au Cambodge, de simples voyageurs dont ils n'étaient pas en mesure de partager la plus anodine des expériences. II apparaît enfin que diverses autres sollicitations, plus directement liées au contexte universitaire ou professionnel, ont également pu participer à la prise de conscience d'une forme de vacance identitaire, que ce soit sous la forme de demandes 
explicites, comme celles formulées lors d'une simulation d'embauche où le candidat était questionné sur son éventuelle capacité à travailler au Cambodge ou encore d'un enseignant sollicitant un travail précis, ou sous forme implicite, en réaction à une opportunité. Ils sont de fait nombreux à avoir présenté un exposé ou soutenu un mémoire portant sur une thématique liée au Cambodge, dans des disciplines aussi variées que la gestion, le commerce ou encore l'architecture.

Le cursus post-baccalauréat a pu ainsi procurer une première opportunité d'approfondir une connaissance sur le Cambodge jusque-là très limitée. Pour certains, cette lente approche s'est finalement concrétisée par un stage de fin d'étude réalisé dans le pays et qui, pour ceux qui sont concernés, a en général constitué la première étape d'une installation durable. En tout état de cause, ce sont semble-t-il ces circonstances qui, fondamentalement, ont commencé à rendre possible l'appropriation et l'objectivation d'une identité qui était jusque-là vécue et parfois subie, mais qui n'était pas à proprement parler conscientisée.

\section{Le départ}

Comme je l'ai mentionné plus haut, il semble que la question du départ ne peut jamais être simplement reliée à un choix parfaitement rationnel et raisonné. II ne me semble pas non plus évident que, comme le suggère Cassarino (2008 : 101), les migrants aient nécessairement et précisément pesé le pour et le contre, comme "the costs and benefits" impliqués par leur décision, d'autant moins d'ailleurs qu'un certain nombre d'entre eux ne savaient pas vraiment à quoi s'attendre. Je crois en revanche que la résolution qui est à l'origine du départ peut plus globalement être mise en perspective avec une forme " d'état identitaire " auquel j'ai tenté de donner corps et qui, dans les circonstances étudiées, pousse plutôt à envisager l'installation au Cambodge comme le résultat d'une forme de manque favorisé par un degré considérable d'assimilation. Le lien entre les deux termes (état identitaire et départ) est complexe, parce que le manque dont il est question n'est qu'un préalable, il n'a pas vocation à tout expliquer. Il établit une condition nécessaire mais pas automatiquement suffisante du départ. II est donc peut-être simplement question d'une aspiration pas toujours consciente, mais par ailleurs très commune parmi les jeunes adultes du pays d'origine, "de découvrir ses racines".

Lorsqu'on s'intéresse de près aux itinéraires, on constate de fait que l'envie de partir pour le Cambodge apparaît bien souvent de façon fortuite, presque soudainement et de surcroît, que la période qui sépare cet instant du moment effectif du départ dépasse rarement quelques mois. On est ainsi poussé à relativiser l'attention portée à la préparation, un sentiment renforcé par le fait que les personnes rencontrées ne possédaient qu'au mieux une expérience sommaire du pays à leur arrivée.

En effet, si certains étaient déjà venus au Cambodge avant leur installation (souvent une seule fois), les circonstances particulières des séjours effectués poussent ici à récuser toute systématisation du lien entre un premier séjour et l'installation définitive. Certains connaissaient ainsi le Cambodge pour y avoir rendu visite à leur père, rentré pour assumer des responsabilités politiques. D'autres étaient venus avec leurs parents pour retrouver des membres de leur famille ayant survécu aux années de guerre. Bien souvent, ces expériences sont antérieures aux années 2000 et le regard porté sur le Cambodge à ce moment-là reste alors celui d'adolescents confrontés à un pays exsangue, gangréné par la pauvreté et la violence : un Cambodge "Far West " où, malgré "la gentillesse des gens", les déplacements étaient rendus compliqués, nécessitant par exemple la présence de gardes du corps. Certains autres ont découvert le pays à un âge plus avancé, en vacances notamment. Ils ne gardent toutefois pas toujours un souvenir plaisant de ce séjour au 
cours duquel ils furent contraints de s'accommoder de la sollicitude des membres de leur famille sur place, souvent perçue comme intrusive, ainsi que d'un sentiment de culpabilité accentué par la rencontre avec des parents aux trajectoires de vie moins privilégiées.

Aujourd'hui encore, les relations familiales continuent de pâtir d'une situation qui est en réalité moins liée à une pression économique ou morale trop importante que la famille locale exercerait sur les migrants (comme la raison la plus immédiate pourrait le laisser supposer) qu'à des acceptions difficilement compatibles des notions d'espace et de vie privés. Ainsi, pour tous ceux qui ont à un moment ou à un autre séjourné chez des parents sur place (y compris chez certains parents de la première génération rentrés depuis la France pour s'installer à Phnom Penh), l'expérience reste marquée par une vraie impuissance à concilier aspirations personnelles et relations de parenté. Cette impossible harmonisation est alors analysée dans les termes d'une altérité culturelle finalement indépassable qui contribue, c'est un fait, à la minceur toujours observée des rapports entre les migrants et leur famille locale. Le témoignage de Puthea, qui en 1998 est venue au Cambodge avec son mari dans le cadre d'un voyage dans trois pays de la région, fournit une lecture intéressante de ces questions et permet de revenir sur la problématique de l'articulation du départ dans l'itinéraire personnel :

" Je ne me voyais pas aller au Vietnam et en Thaillande et ne pas mettre les pieds au Cambodge. Je me disais, quand même, il faut que j'aille voir ce que c'est. C'était l'Asie quand même. J'avais envie de connaître l'Asie. Mais en revenant, même pas envie, parce que j'étais bien en France, tu vois on avait une vie tout à fait... Qui nous plaisait, on était Parisiens, on avait nos enfants, nos amis,

notre boulot, pourquoi bouger?

[à propos de leur semaine passée au Cambodge :]

C'était très intense, c'était... on est venu en 98 et... et donc c'était encore à l'époque... Mon oncle, qui était en vacances, un des frères de mon père, qui habitait en France, parce que lui est venu beaucoup plus tôt que mon père et a acheté une maison rapidement... donc il était en vacances à ce moment-là et donc on a dormi chez lui à Toul Kork. Et c'était encore à l'époque où, enfin, très peu de rues étaient pavées, où on était à Toul Kork, enfermés à clé, on ne sortait pas le soir. Et, je savais même pas qu'il y avait une vie où les expatriés pouvaient vivre. On a été pris par la famille, c'était très étouffant... Le seul moment libre c'est quand on est parti tous les deux à Siam Reap, et encore ils voulaient nous accompagner. Où... Où c'était. Oui quand on est venu ici, c'était encore l'insécurité et puis, surtout le fait d'être chez des Cambodgiens... Ils avaient vachement peur pour nous. Ils avaient une responsabilité tu vois, ils recevaient quand même la nièce et son mari français donc il fallait que tout se passe bien. Et on a eu une vision d'un Cambodge... Côté cambodgien, local, mais on était très protégé, on allait dans les endroits qu'il fallait. On n'a jamais fait, on n'est jamais sorti prendre un verre. On savait même pas que c'était possible... [...]. Après, je me suis dit: "je reviendrai avec mes enfants en vacances" mais jamais dans l'idée... en me disant c'est mon pays, et je m'y installe, j'ai envie de le connaître. Et le déclic est venu deux trois ans plus tard mais... comme une envie d'une bière quoi ".

$\mathrm{Si}$, donc, pour certains, le départ s'est inscrit dans un itinéraire rectiligne, comme point d'orgue d'un parcours universitaire par exemple, ils sont nombreux à avoir dû abandonner une situation professionnelle confortable pour partir. Certains autres ont eu la chance de pouvoir bénéficier d'une mise à disposition ou de trouver un emploi depuis la France. À une exception près, aucun ne possédait une expérience approfondie du pays au moment de son départ et beaucoup n'en maîtrisaient même pas la langue (même si l'apprentissage passif réalisé au contact des parents leur a ensuite largement facilité la tâche). Un seul avait réalisé au préalable un voyage de reconnaissance avec pour objectif de s'installer. Tous les autres ont choisi de partir en réponse à une opportunité, un besoin ou une (im)pulsion. 


\section{À propos de l'arrivée au Cambodge. Le cogito réflexif et la fin d'un cycle identitaire}

En définitive, la présentation qui a été réalisée jusqu'ici, bien qu'elle n'ait pu rendre compte de toute la variété et la complexité des itinéraires ni de chacune des étapes clés qui ont été évoquées, a je crois permis de justifier quelque peu le lien entre une assimilation française réussie (au sens où l'entendent les institutions du pays) et le départ. Dans cette perspective, c'est donc presque toujours une profonde méconnaissance du Cambodge, que j'ai qualifiée de vacance identitaire, qui constitue en réalité la toile de fond du départ. C'est enfin de ce contexte, de cet "état ", que l'idée d'un départ semble à un moment s'être formulée puis détachée, comme l'aboutissement d'une maturation dont les racines sont profondes, ancrées au plus loin de l'enfance et même de la généalogie.

Paradoxalement, ce manque identitaire fondateur semble intimement associé à une forme d'accomplissement, impliquée par la validation de critères structurels propres à la société d'origine (française en l'occurrence). C'est également ce qu'incitent à penser certains témoignages qui évoquent le défaut de " confiance " de ceux qui ne font jamais le pas de venir s'installer durablement (mais que cela n'empêche pas de venir fréquemment pour des séjours ponctuels), une confiance que d'autres acquièrent (en France) par adéquation avec le modèle imposé d'immigration, et qui assurerait de ne pas perdre ce qui a été obtenu. Les sentiments d'appartenance et de reconnaissance sont sécurisants, c'est un fait. Ils semblent conférer de la linéarité aux parcours en permettant une construction par étape qui, à ce titre, ne nécessite que très peu d'aller-retour. Au sens le plus concret, on peut supposer que cela se traduit, chez les migrants de l'enquête, par une sédentarité très marquée et un transnationalisme peu significatif, voire pratiquement nul. Cela ne signifie pas que les parcours transnationaux souvent décrits dans la littérature scientifique sont nécessairement liés à un manque de confiance, de reconnaissance de la société d'origine, mais il me semble qu'il y a là un lien qui n'est pas anodin et qui reste pourtant largement négligé. On constate en effet que si de nombreux auteurs s'attachent à montrer la diversité des formes d'adaptation aux contraintes structurelles du pays d'accueil (par exemple Glick-Shiller et al., 2005), ils n'évoquent que plus rarement le ressenti des personnes concernées, préférant ne voir dans leur créativité qu'une forme de résistance idéologique (dont on se demande parfois à qui, de l'observateur ou de ses informateurs elle appartient) plutôt, par exemple, qu'un simple ajustement à des contraintes intimes objectives.

Pour reprendre le fil de l'argumentation et forcer délibérément le trait, on serait donc tenté d'écrire que les personnes avec qui j'ai réalisé cette recherche ne sont pas issues d'un entre-deux en ce sens qu'elles ont grandi et se sont développées sans prêter attention à ce positionnement. Si l'on se place dans la perspective de François Laplantine et Alexis Nouss (1997), ce dernier n'est au reste que relatif, car nous sommes finalement tous issus d'un processus immuable de métissage qui, pourrait-on en revanche ajouter, s'essentialise ou se coagule au gré des contextes, des influences ou encore, bien entendu, du bon vouloir. Dans cette hypothèse, $c^{\prime}$ est seulement lorsqu'il commence à être isolé par la conscience, puis formulé, que le positionnement à l'entre-deux s'impose comme une essence qu'il est alors possible de s'approprier et sur la base de laquelle un discours plus ou moins politique pourra finalement se développer.

C'est en tout état de cause sur cet aspect sensible de l'identité que je voudrais à nouveau insister ici (après l'avoir évoqué lorsqu'il était question de la perception des migrants par les Cambodgiens), car la période qui suit l'arrivée au Cambodge contribue par réaction à l'essentialisation de tout un pan identitaire, au passage à l'état réflexif d'une identité cambodgienne jusque-là largement cantonnée à un niveau pré-réflexif. Tous 
mes informateurs évoquent ainsi l'étrange sensation et souvent la jubilation qu'ils ont éprouvées lors de leur première plongée dans le bain de stimuli à la fois très exotiques et pourtant souvent familiers que provoque l'arrivée au Cambodge. La reconnaissance et I'identification qui s'opèrent alors n'ont rien de réfléchi. Elles sont impliquées et imposées par les diverses intrusions sensorielles auxquelles les migrants sont exposés.

La langue est peut-être le plus évident de ces stimuli, parce qu'elle se trouve immédiatement parlée par tous et partout. Elle fait alors écho à des domaines précis de la vie des personnes (familiaux, communautaires), portant un premier éclairage, le moins spécifique, sur l'existence d'une identité cambodgienne latente. Certains sont ensuite interpellés par des souvenirs plus précis, plus personnels, notamment des odeurs de cuisine, de condiments, mais aussi des ambiances olfactives comme celle, très spécifique, qui accompagne la pluie et dont une informatrice ayant quitté le Cambodge à un âge avancé pensait avoir gardé la trace. À ces éléments s'ajoute ensuite la reconnaissance plus diffuse de contextes sociaux et de la nébuleuse sensible qui leur est associée. Les ambiances de fête, l'atmosphère particulière qui caractérise les préparations de repas ou encore des "discussions entre hommes" renvoient toutes à des moments de l'enfance et de l'adolescence qu'elles lient par la force des choses à une réalité cambodgienne. Au contact des Cambodgiens, certains prennent également conscience de l'apprentissage corporel qu'ils ont malgré tout réalisé et qui se révèle en général de lui-même lorsqu'il faut interagir dans des circonstances particulières de la vie sociale où les attitudes corporelles sont particulièrement contrôlées (comme les diverses fêtes par exemple). D'autres découvrent enfin chez leurs hôtes des traits de caractère (notamment des formes de timidité) qu'ils pensaient très personnels mais qui correspondent, au Cambodge, à une certaine normalité. Le recensement de ces " détails " qui, par association avec des traces sensorielles, font finalement apparaître, par surimpression, une forme de cambodgénéité jusqu'alors latente, devrait probablement être systématisé. II devrait aussi être mis en regard de l'apprentissage plus pragmatique qui se nourrit des rencontres, surtout lorsqu'elles contribuent à éclaircir l'histoire familiale en donnant matière au silence des parents et à leurs choix d'éducation. Il reste néanmoins que l'individu est, au cours de cette période, placé au cœur d'un pétillement sensoriel (pour ne pas dire sémiotique) qui déborde largement sa conscience et par lequel il ne se contente pas de produire du sens, mais fait lui-même sens. Ce processus, qui fait " se sentir vivant " selon les mots d'un informateur, est bien entendu limité dans le temps parce que le jeu d'association " naturelle " des signes, qui donne une dimension superlative au cogito cartésien (je suis parce que je pense), ne peut s'opérer éternellement. S'ensuit une période d'apaisement puis un "retour à la normale " qui ne l'est plus tout à fait parce que, bien souvent, la question de "l'entre-deux " a été appropriée et que se pose désormais la question d'un positionnement qui n'est plus seulement sensible, mais conscient et parfois même politique.

Cette période, qui dure en général quelques mois, marque donc une étape forte dans le processus identitaire que j'ai à plusieurs reprises évoqué parce qu'elle amène les personnes à mieux comprendre leurs parcours, certains de leurs traits de caractère ou encore parce qu'elle contribue à leur faire prendre conscience des avantages et des inconvénients associés à l'essentialisation de leur identité cambodgienne. On voit alors apparaître certains " besoins " qui ne s'étaient que plus rarement manifestés en France, comme par exemple celui de côtoyer d'autres Franco-Cambodgiens, alter ego à la spécificité maintenant bien reconnue et seuls susceptibles de partager la connivence qui découle du fait de pouvoir porter "simultanément un regard français sur les Cambodgiens et un regard cambodgien sur les Français ". C'est également souvent dans la période qui suit que l'idée de l'accomplissement de ce qui est présenté comme " une quête identitaire " se développe, laissant la place à une normalisation du quotidien qui fait que la 
grande majorité des migrants de l'étude ressemblaient à bien des égards à la plupart des expatriés français. Pour beaucoup aussi, leur histoire avec le Cambodge pouvait désormais se dénouer à tout moment, parce qu'ils n'étaient quasiment plus retenus que par des considérations matérielles.

\section{Conclusion}

Cet article n'avait pas pour objectif (est-il besoin de le préciser ?) de minimiser l'importance des explications rationnelles souvent associées aux migrations et à la question des identités en leur opposant une réduction tout aussi stérile au sensible. Considérant la prégnance historique du présupposé analytique cartésien sur les autres formes de pensée (cf. à propos du don Mariani, 2011), il me semble néanmoins opportun d'insister ici sur ce qui ne se conçoit pas, et de chercher ainsi à replacer l'esprit dans son corps en se positionnant de la sorte dans une perspective phénoménologique, fondée sur le principe que "l'existence précède l'essence ". Comme on peut également l'établir dans les termes de la psychologie freudienne, le " contenu manifeste " des identités n'est qu'une réalisation possible d'un " contenu latent " complexe et diffus. Dans cette hypothèse, cet article est un préalable, car son objectif principal était de donner quelques-uns des éléments d'existence parmi lesquels les personnes rencontrées s'essentialisent, plus ou moins, en différents instants et en différents endroits. II n'y a donc aucune exclusivité dans cette démarche, qui n'empêche pas qu'on s'intéresse plus tard aux stratégies et autres calculs qui se développent sur la base des éléments sensibles, relevant donc d'une forme de " conscience irréfléchie ", qui dessinent la trame de fond avec (plutôt que sur) laquelle les personnes se meuvent dans l'univers social.

Finalement, il ressort que cette trame épouse à s'y méprendre les contours de la progression classique de l'individualité dans la société française (ce qui est en soi un argument susceptible d'étayer le point de vue des migrants sur la "réussite " de leur intégration) et que l'histoire de la migration ne peut donc être ici séparée de celle de la construction de la personne, intimement et socialement située. D'où le choix de parler " d'états identitaires " incitateurs, autant de configurations personnelles complexes mais non explicitées qui sont propices au départ puis à un certain type d'installation. Il convient en effet d'insister sur la sédentarité des personnes rencontrées, un caractère qui ne présage pas forcément un enracinement définitif, mais qui correspond à un état d'esprit par définition incompatible avec le mode de vie multi-situé qui, si on se fie à l'abondante littérature sur le sujet, semble être celui des migrants transnationaux par exemple. II y aurait donc un lien à creuser entre le développement de la personne et son mode d'être au monde, avec cette idée que les choses ne se font pas, dans le cas présent, de façon synchronique, sur plusieurs fronts simultanés, mais de manière diachronique, par étapes et enfin, qu'une telle particularité est aussi la conséquence d'un certain modèle d'intégration. Je souhaiterais ainsi faire l'hypothèse que plus les individus sont assimilés au modèle français moins ils sont susceptibles de s'inscrire dans une perspective transnationale (mais que cela ne réduit pas nécessairement leur volonté de départ, au contraire même). II serait nécessaire, en outre, de mieux situer la population de cette étude dans I'ensemble de la population migrante de Franco-Cambodgiens afin de vérifier si, comme je le pense, les migrants de cette étude constituent bien, au sein de la seconde génération installée à Phnom Penh, une population majoritaire. Quoi qu'il en soit de la réalité de cette impression, il y a là une catégorie de migrants qui reste largement ignorée, notamment parce que les travaux portant sur les Franco-Cambodgiens en France ont tous ou presque pour cadre de grandes agglomérations (Rennes et Lyon notamment) ou des lieux de concentration importante. A contrario, la présente étude révèle des origines disparates ainsi que le rôle clé que cette distribution géographique semble jouer dans le phénomène. 
En définitive, on pourrait avancer que malgré leur positionnement constructiviste, adopté en réaction à un essentialisme qu'elles dénonçaient, les transnational studies semblent avoir persisté à propager le mal qu'elles combattaient. Car en effet, comment ne pas voir qu'en instaurant le caractère transnational de certains migrants en élément fondateur des "nouvelles " migrations, elles ont à leur tour fini par imposer une singularité comme un universalisme. Du même coup, il a été omis que "l'entre-deux " est aussi un construit social, un objet relationnel, produit de la conscience de l'observateur. Une manière alternative d'envisager la question consisterait ainsi à se demander, à chaque fois, à quel moment et pour qui (et pourquoi), de l'immigré, du migrant, de l'État, du sens commun et/ou du chercheur, cet entre-deux (ou trois ou quatre) le devient-il ? Une telle démarche permettrait de rendre justice aux objets en les replaçant dans le contexte relationnel dont ils sont les produits plutôt que d'en isoler certaines dimensions seules, où se confondent le principe de la pensée et l'objet de la pensée (Merleau-Ponty, 1964 : 73). Dès lors, on pourrait reformuler les termes du problème en ouvrant une comparaison approfondie entre ceux qui, comme la majorité des migrants de mon enquête, ont pu ou dû s'approprier cet entre-deux à l'âge adulte et ceux qui l'éprouvent depuis toujours, dans des formes plus ou moins institutionnalisées. Plus généralement, on s'intéresserait donc aux circonstances dans lesquelles les choses commencent à être nommées et à l'influence de cette classification sur la réalité.

Dans les circonstances de la relation d'enquête, cela implique de prendre en compte que la construction du sens est tributaire des capacités sémiotiques de chacun comme de I'horizon constitué par les discours légitimes préexistants, prisme auquel l'expérience personnelle ne peut manquer d'être passée bien qu'elle le déborde systématiquement. Comme l'observateur, les informateurs possèdent des critères de compréhension hérités de leur histoire, de leurs rencontres, mais aussi des diverses interprétations auxquelles ils ont préalablement eu accès. La question, pour l'enquêteur et pour son informateur, est donc de trouver les mots et les catégories les plus appropriés dans un univers très largement pré-produit de discours et de concepts à l'influence variable qui, inévitablement, orientent les conversations et la perception. Dans ces circonstances, l'objectif spécifique de l'ethnologue qui travaille à partir d'histoires de vies peut être d'aider son interlocuteur à trouver un mode d'expression qui soit le plus personnel possible.

\section{Références bibliographiques}

Bourdieu Pierre (1986) L'illusion biographique, Actes de la recherche en sciences sociales, 62-63, pp. 69-72.

Bretell Caroline (2003) Anthropology and Migration: Essays on Transnationalism, Ethnicity and Identity, Walnut Creek, Altamira Press, 239 p.

Candau Joël (2011) Une théorie sensorielle de I'identité, in Paul-Louis Colon Éd., Le sens et les sciences sociales, Paris, éditions Petra.

Cassarino Jean-Pierre (2008) The Conditions of Modern Return Migrants, International Journal on Multicultural Societies, 10 (2), pp. 95-105.

Cerase Francesco (1974) Expectations and Reality: A Case Study of Return Migration from the United States to Southern Italy, International Migration Review, 8 (2), pp. 245-262. 
Glick Schiller Nina, Nieswand Boris, Schlee Günther, Darieva Tsypylma, Yalcin-Heckmann Lale and Fosztó Lásló (2005) Pathways of Migrant Incorporation in Germany, Transit, 1 (1), [en ligne]. URL : http://escholarship.org/uc/item/90b8w0dh

Guillou Anne-Yvonne (2001) Postures et apparence physique khmères, du Cambodge à la France, Hommes et migrations, 1234, pp. 90-94.

Levitt Peggy and Waters Marie C. (Eds.) (2002) The Changing Face of Home: The Transnational Lives of the Second Generation, New York, Russell Sage Foundation, 408 p.

Herzfeld Mickael (1992) La pratique des stéréotypes, L’Homme, 121, pp. 67-77.

Laplantine François et Nouss Alexis (1997) Le Métissage, Paris, Flammarion, 127 p.

Lévi-Strauss Claude (1971) L’Homme nu (Mythologiques, 4), Paris, Plon, 688 p.

Mariani Léo (2011) Pouvoir et société. Repenser le lien social dans le Laos urbain, Anthropos, 106 (2), pp. 397-409.

Mauss Marcel (1936) Les techniques du corps, Journal de psychologie, 32 (3-4), pp. 363-386.

Merleau-Ponty Maurice (1964) Le visible et l'invisible, Paris, Gallimard, 360 p.

Nann Stéphanie (2009) Les Familles Cambodgiennes en France : histories de vie et reconstruction, Dialogue, 185 (3), pp. 55-66.

Nann Stéphanie (2007) Les Cambodgiens en France, entre l'image et la réalité, Migrations Société, (19) 109, pp. 147-162.

Phong Dang, Husson Laurence et Charbit Yves (2000) La diaspora vietnamienne : retour et intégration au Vietnam, Revue Européenne de Migrations Internationales, 16 (1), pp. 183-205.

Ricœur Paul (1985) Temps et récits III, Le temps raconté, Paris, éditions du Seuil, 538 p.

Simon-Barouh Ida (1989) Les Cambodgiens en France : une identité retrouvée et transformée, Migrants-Formation, 76, pp. 67-79.

Souvannavong S.-A. S. (1997) Elites in exile. The Emergence of aTransnational Lao Culture, in Grant Evans Ed., Laos, Culture and Society, Bangkok, Silkworm Books, pp. 100-124. 


\section{Léo Mariani}

\section{Identités narratives et identifications sensibles : itinéraires de migrants franco-cambodgiens de seconde génération à Phnom Penh}

Le présent article expose les résultats préliminaires d'une recherche réalisée dans la capitale cambodgienne, Phnom Penh. L'objectif principal était de mieux appréhender les itinéraires de certains migrants franco-cambodgiens " de retour ", appartenant en réalité à la deuxième génération et donc nés en France où y ayant passé l'essentiel de leur vie. De manière intéressante, la plupart des arguments qui servent habituellement à expliquer les migrations, comme la volonté d'aider, de profiter d'opportunités professionnelles ou encore l'influence des réseaux sociaux ou le mythe du pays d'origine, ne semblent pas pertinents dans le cas de la population étudiée. L'argumentation insiste en revanche sur le processus identitaire qui constitue dans chaque cas la toile de fond de la migration, reliant cette caractéristique à I'histoire du Cambodge, à certaines caractéristiques structurelles du pays d'origine et aux formes d'intégration observées sur place.

\section{Narrative Identities and Sensitive Identifications. A Reconstruction of some French Cambodian Second Generation Migrants' Itineraries in Phnom Penh}

This article states the first results of a survey conducted in Phnom Penh, the Cambodian capital city. The main aim of the research is the understanding of second generation French Cambodian remigration processes. Remarkably, classical arguments explaining migrations, such as the willingness to help, opportunities for making money, network influence, myth of the country of origin, seem less relevant for this group. Alternatively, this study argues that questions of identity are at the background of remigration decision and relates them to Cambodia's specific history, contextual parameters in the hosting country, France, and specificities of the shapes of integration observed in Phnom Penh.

\section{Identidades narrativas e identificación sensible. Reconstrucción de las rutas de migrantes franceses de Camboya de segunda generación en Phnom Penh}

Este artículo expone los resultados de un estudio hecho en la capital camboyana, Phnom Penh. El objetivo principal ha sido de aprehender los itinerarios de algunos repatriados, Francés de Camboya que regresan al país. Ellos pertenecen a la segunda generación, la que nació en Francia o la que vivió la mayoría de su vida allí. De manera muy interesante, la mayoría de los argumentos que sirven generalmente para explicar el fenómeno de las migraciones, como por ejemplo, la voluntad de ayudar a la gente del país de origen o de aprovechar de las oportunidades profesionales o del predomino de los redes sociales no parecen tener eco en la población estudiada. Al contrario, la argumentación insiste más en el proceso identitario, siempre evidente dentro del fenómeno de migración, relacionando esta especificidad a la historia de Camboya y a algunas características estructurales del país de origen pero también a los diferentes tipos de integración observados en el sitio. 